Algebraic $\& \mathcal{G}$ Geometric Topology

Volume 1 (2001) 321-347

Published: 31 May 2001

ATG

\title{
On McMullen's and other inequalities for the Thurston norm of link complements
}

\author{
Oliver T. Dasbach \\ BRIAN S. MANGUM
}

\begin{abstract}
In a recent paper, McMullen showed an inequality between the Thurston norm and the Alexander norm of a 3-manifold. This generalizes the well-known fact that twice the genus of a knot is bounded from below by the degree of the Alexander polynomial.

We extend the Bennequin inequality for links to an inequality for all points of the Thurston norm, if the manifold is a link complement. We compare these two inequalities on two classes of closed braids.

In an additional section we discuss a conjectured inequality due to Morton for certain points of the Thurston norm. We prove Morton's conjecture for closed 3 -braids.
\end{abstract}

AMS Classification 57M25; 57M27, 57M50

Keywords Thurston norm, Alexander norm, multivariable Alexander polynomial, fibred links, positive braids, Bennequin's inequality, Bennequin surface, Morton's conjecture

Recently, the multivariable Alexander polynomial experienced a fulminant comeback on the mathematical stage. Connections to Donaldson-Seiberg-Witten theory and also its meaning in terms of Vassiliev knot invariants became known. In this paper we focus our attention on the recent inequality of McMullen [McM99] that compares the Alexander norm of a 3-manifold $M$ with its Thurston norm. Both norms are norms on $H^{1}(M ; \mathbb{R})$. If the manifold is the complement of an oriented link $L$ in $S^{3}$ or, more generally, in an integral homology sphere then the meridians of $L$ give a natural base for $H^{1}(M, \mathbb{R})$. The Thurston norm at the point $(1, \ldots, 1)$ is determined by a minimal spanning surface of the link. McMullen shows that if the first Betti number of the manifold is at least 2 then the Thurston norm is greater than or equal to the Alexander norm on all points. Moreover, he compares the value on all complements of links with less than 9 crossings. McMullen's results extend the well-known fact that twice the genus of a knot is greater than or equal to the span of the Alexander polynomial. 
On the other hand, another inequality for the Thurston norm of link complements, evaluated at certain points, is sometimes quite useful: The Bennequin inequality. We will extend this inequality to an inequality for all points of the Thurston norm.

To show the differences, we will compare these two inequalities on two classes of links.

(1) The first class is the class of closed homogeneous braids, i.e. braids in which the standard generator $\sigma_{i}$ appears at least once for every $i$, and all exponents of $\sigma_{i}$ have the same sign. By a result of Stallings [Sta78] these links are known to be fibred and the fibre surface is the one that one gets by applying Seifert's algorithm.

This implies that the Thurston and the Alexander norm of the complements of these links agree at the points given by the fibration. With some additional arguments we will give the equality for all points $C=$ $\left(C_{1}, \ldots, C_{r}\right) \in H^{1}(M, \mathbb{R})$ with $C_{i} \geq 0$. In contrast to this result, Dunfield [Dun99] shows that the equality does not necessarily hold at all points for three-manifolds that fibre over the circle.

The Bennequin inequality is in general not helpful for this class of links. We will give, however, a simple proof of a recent result [Kan98] saying that the difference between the Bennequin number and the Thurston norm can be arbitrarily large.

(2) The second class is the class of closed band positive braids. The band generators, a new set of generators of the braid groups, have recently appeared quite useful [BKL98]. One defines $a_{i, j}$ to be the braid

$$
\sigma_{i}^{-1} \cdots \sigma_{j-2}^{-1} \sigma_{j-1} \sigma_{j-2} \cdots \sigma_{i} \text {. }
$$

With these band generators, band positive braids are braids where all $a_{i, j}$ appear only with positive exponents.

Ko and Lee [KL97] showed that a band Seifert algorithm for closed band positive braids on four strands yields a minimal spanning surface. By computing the Alexander polynomial of an example they point out that furthermore these band positive braids are not necessary fibred. From this example and others, it follows that McMullen's inequality is not sharp for this class of links.

The Bennequin inequality gives the Thurston norm at the point $C=$ $(1, \ldots, 1)$ for closed band positive braids of arbitrary braid index. In particular the result of Ko and Lee holds for an arbitrary number of strands. It follows that there is a Bennequin surface, in the sense of [BM91], for every closed band positive braid. For special classes of band 
positive braids, the generalization of Bennequin's inequality will give us the Thurston norm at all points $C=\left(C_{1}, \ldots, C_{r}\right), C_{i} \geq 0$.

In addition to these two inequalities there is a conjectured inequality, due to Morton, for points $C=\left(C_{1}, \ldots, C_{r}\right),\left|C_{i}\right|=1$, of the Thuston norm that comes from the HOMFLY polynomial. This inequality would sharpen the Bennequin inequality.

To prove it, however, seems to be a hard task. It is known that this conjecture is true for all closed homogeneous braids. We will give a new and different proof of this result, and we will prove that it is also true for the class of closed 3-braids.

Our proof of this latter result uses powerful tools, as a beautiful theorem of Scharlemann and Thompson, based on Gabai's work.

Added in proof: It was well-known, that the span of the Alexander polynomial does not give a lower bound for the slice-genus of a knot. The slice-genus is the minimal genus of an oriented smooth embedded surface $F$ in $D^{4}$ with boundary the knot. Recently, Ferrand [Fer00] gave an example of a knot where a "slice Morton conjecture" does not hold. The slice Bennequin inequality, however, was proven in [Rud93].

Acknowledgment The work to this paper was mainly done while the first author was visiting Columbia University in Summer 1999. The authors would like to express their deep gratitude to Joan Birman for many helpful discussions and suggestions that influenced this paper. We also appreciate very much the efforts of an anonymous referee to make the paper more readable.

Furthermore, the first author thanks Hugh Morton, Guennadi Noskov and Arkady Vaintrob for their helpful remarks.

The work of the first author was partially supported by the Deutsche Forschungsgemeinschaft (DFG).

\section{Setting the scene}

\subsection{A generalization of Seifert's algorithm}

By a classical result of Seifert, every oriented link is the boundary of an oriented surface in $S^{3}$. His algorithm to construct such a surface is by smoothing every 
crossing in a diagram of the link (see Figure 1). This gives a collection of circles, called Seifert circles. Now bands are added to join the Seifert circles meeting at a crossing. The surface so constructed has Euler characteristic $(s-c)$ where $c$ is the number of crossings and $s$ is the number of Seifert circles.

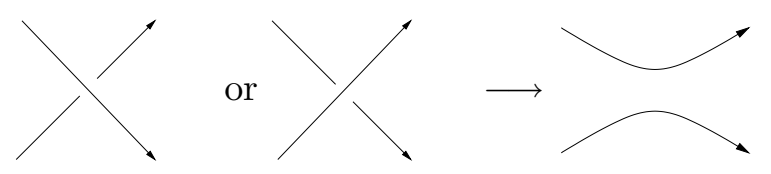

Figure 1: Smoothing a crossing

One can generalize Seifert's algorithm to find a punctured oriented surface spanning a sublink of a link in the complement of the other components: For the sublink construct a surface as described above. Whenever another component is undercrossing a component of the sublink we get a piercing of the surface.

By a minimal spanning surface for a link we always mean an oriented surface in $S^{3}$, without closed simply connected components, of maximal Euler characteristic that has the link as its boundary. This surface need not be connected.

\subsection{The Thurston norm}

Let $M$ be a 3 -manifold and $b_{i}$ the $i$-th Betti number $M$, i.e. the rank of $H_{i}(M ; \mathbb{R})$. In $\left[\right.$ Thu86] Thurston defined a semi-norm $\|\cdot\|_{T}$ on $H^{1}(M ; \mathbb{R})$. For most interesting cases, e.g. for hyperbolic manifolds, this semi-norm is actually a norm.

Given a properly embedded connected surface $S$ in $M$ let

$$
\chi_{-}(S):=\max \{-\chi(S), 0\},
$$

where $\chi(S)$ is the Euler characteristic of $S$. If $S$ is not connected then $\chi_{-}(S)$ is defined to be the sum of $\chi_{-}\left(S_{i}\right)$ over each connected component $S_{i}$ of $S$.

Given a class $C$ in $H^{1}(M ; \mathbb{Z})$ define $\|C\|_{T}:=\min \left\{\chi_{-}(S)\right\}$ where $S$ is a properly embedded oriented surface which represents the dual to $C$ in $H_{2}(M, \partial M ; \mathbb{Z})$. The definition extends uniquely to a semi-norm on $H^{1}(M ; \mathbb{R})$. This seminorm is symmetric about the origin and the unit-ball is a convex finite-sided polyhedron with rational vertices. If $\chi_{-}(S)$ equals the Thurston norm of the homology class $C$ of $S$ then we say that $S$ is Thurston norm minimizing for $C$. Gabai [Gab83] showed that one can relax the requirement for the surfaces to be properly embedded and work with arbitrary images of surfaces instead. 
We are mainly interested in 3-manifolds that are link complements in $S^{3}$. For a smoothly embedded oriented link $L=L_{1} \cup \cdots \cup L_{r} \subset S^{3}$ with $r$ components, let $M=S^{3}-\mathcal{N}(L)$ be the compact 3-manifold obtained by deleting a tubular neighborhood $\mathcal{N}(L)$ of $L$. The orientation of $L$ induces an orientation on the meridians (see Figure 2) and thus, after choosing an ordering of the components, a multiplicative basis $\left\langle t_{1}, \ldots, t_{r}\right\rangle$ for $H_{1}(M ; \mathbb{Z}) \cong a b\left(\pi_{1}(M)\right)$.

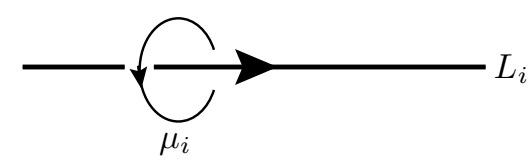

Figure 2: Orientation of the meridians

Let $\mu_{i}$ be a meridian curve in $\partial \mathcal{N}\left(L_{i}\right)$ representing $t_{i}$. For a class $C=$ $\left(C_{1}, \ldots, C_{r}\right) \in H^{1}(M ; \mathbb{Z})$, with respect to the basis dual to the $t_{i}$, we get:

Lemma 1.2.1 There exists a Thurston norm minimizing surface $S$ for the class $C$ such that $S$ is a properly embedded oriented surface in $M$ with $S \cap$ $\partial \mathcal{N}\left(L_{i}\right)$ a collection $\gamma_{i}=\left\{\gamma_{i_{1}}, \ldots, \gamma_{i_{k}}\right\}$ of parallel simple closed curves, essential in $\partial \mathcal{N}\left(L_{i}\right)$, such that $\left|\mu_{i} \cap \gamma_{i}\right|=\left|C_{i}\right|$.

Proof That there exists a properly embedded oriented norm minimizing surface $S$ for any class $C \in H^{1}(M ; \mathbb{Z})$ is explained in [Thu86]. It remains to show that we may arrange to have $\partial S$ as indicated.

We may assume that $S$ is incompressible and boundary incompressible, since compressions do not change the homology class of the surface and do not raise the value of $\chi_{-}(S)$. So, since $\partial M$ is a collection of tori, $S$ has $S \cap \partial \mathcal{N}\left(L_{i}\right)$ consisting of some number (possibly zero) of parallel simple closed curves, essential in $\partial \mathcal{N}\left(L_{i}\right)$.

If there are two components of $\gamma_{i}$ with opposite orientations, then there is an innermost pair of such components. That is, there are two components $\gamma_{i_{1}}$ and $\gamma_{i_{2}}$ (without loss of generality) with opposite orientations such that $\gamma_{i_{1}} \cup \gamma_{i_{2}}$ is the boundary of an annulus $A$ in $\partial \mathcal{N}\left(L_{i}\right)$ with $\gamma_{i} \cap \operatorname{int}(A)$ empty. We can alter the surface $S$ by attaching a copy of the annulus $A$ to $S$ and pushing it into the interior of $M$. This operation does not change the homology class of $S$ or $\chi_{-}(S)$. By repeatedly applying this procedure, we arrange that every component of $S \cap \partial \mathcal{N}\left(L_{i}\right)$ has the same orientation.

Now homotop $\mu_{i}$ so that $\mu_{i} \cap \gamma_{i}$ is minimal. Then the absolute value of the algebraic intersection number of $\mu_{i}$ and $\gamma_{i}$ equals the number of points of intersection of $\mu_{i}$ and $\gamma_{i}$. That is, $\left|C_{i}\right|=\left|\mu_{i} \cap \gamma_{i}\right|$. 


\subsection{The Alexander norm and McMullen's inequality}

To get an estimate for the Thurston norm, McMullen defined a norm which is related to a classical invariant in knot theory: The Alexander norm $\|\cdot\|_{A}$ on $H^{1}(M ; \mathbb{Z})$. For this, one looks at the multivariable Alexander polynomial for a 3 -manifold [Tur75]. For $t_{1}, \ldots, t_{r}$ a set of generators of $H_{1}(M ; \mathbb{Z}) /($ torsion), it is a polynomial $\Delta_{M}$ in $\mathbb{Z}\left[t_{1}, \ldots, t_{n}\right]$. We are only dealing with link complements, so the $t_{i}$ can be assumed to be homology classes of the meridians of the link.

For a class $C \in H^{1}(M ; \mathbb{Z}) \cong \operatorname{Hom}\left(H_{1}(M ; \mathbb{Z}), \mathbb{Z}\right)$, the Alexander norm of $C$ is defined to be $\|C\|_{A}:=\sup C(P-Q)$ where $P$ and $Q$ ranges over those monomials $t_{j_{1}} \cdots t_{j_{l}}$ which appear with non-zero coefficients in the Alexander polynomial of $M$. Again, this norm is sometimes only a semi-norm.

McMullen [McM99] proved an inequality for the Thurston norm in terms of the Alexander norm. For all $C \in H^{1}(M ; \mathbb{Z}), M$ a link complement,

$$
\|C\|_{A} \leq\|C\|_{T} \quad \text { for } b_{1}(M) \geq 2 .
$$

If $M$ is a knot complement and $C$ the generator of $H^{1}(M)$ we have:

$$
\|C\|_{A} \leq\|C\|_{T}+1 \text {. }
$$

Inequality (2) is the well-known fact that the span of the Alexander polynomial of a knot is less than or equal to twice its genus. If $M$ is not a link complement then one has to take the third Betti number into account in Inequality (2) [McM99].

\subsection{Bennequin's inequality}

There is another, well-known, inequality for the Thurston norm at certain points in $H^{1}(M ; \mathbb{R})$. In the sequel of this section, we will extend it to all points. Let $\mathfrak{b} \in B_{n}$ be a braid that closes to a link $L$, where the orientation is so that all strands are oriented in the same direction.

The Bennequin number $\beta_{t}$ for $\mathfrak{b}$ is defined to be

$$
\beta_{t}(\mathfrak{b}):=\operatorname{pos}(\mathfrak{b})-n e g(\mathfrak{b})-n
$$

where $\operatorname{pos}(\mathfrak{b})$ is the number of positive crossings and $n e g(\mathfrak{b})$ the number of negative crossings (see Figure 3 ). The difference $\operatorname{pos}(\mathfrak{b})-n e g(\mathfrak{b})$ is also called algebraic crossing number. (The $t$ in $\beta_{t}$ stands for "transversal" since it is closely related to and has its origins in transversal knot theory, see e.g. [FT97, BW99].) 


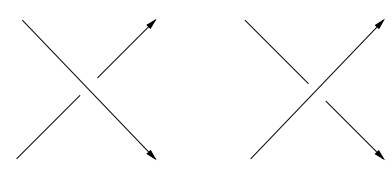

Figure 3: Positive and negative crossing

Since, by the algorithm of Pierre Vogel [Vog90], every link can be transformed into a closed braid just by Reidemeister II moves without changing the number of Seifert circles, this definition extends to arbitrary link diagrams by replacing $n$ by the number of Seifert circles in the link diagram.

Let $S$ be an oriented Seifert surface without closed connected components that spans the closure of $\mathfrak{b}$ and maximizes the Euler characteristic $\chi$.

In his thesis and the paper thereafter [Ben83] Bennequin proved:

Theorem 1.4.1 (Bennequin's inequality) $\beta_{t}(\mathfrak{b}) \leq-\chi(S)$.

By definition, $-\chi(S) \leq \chi_{-}(S)$ for a surface $S$. Hence, Bennequin's inequality gives us a lower bound for the Thurston norm at the points $C=\left\{C_{1}, \ldots, C_{r}\right\}$ with $\left|C_{i}\right|=1$ for all $i$.

Note that the Bennequin number depends on the form of the realization of the link as a closed braid and is not an invariant of the link itself. The question of finding the maximal Bennequin number among all representatives of a given link type seems to be harder. We will come back to this problem in Section 4.

\subsubsection{Relative Bennequin number}

For a link, given as a closed braid or as a diagram, the Bennequin number gives a lower bound for the Thurston norm of a single cohomology class. The Bennequin numbers for sublinks give a much weaker lower bound for corresponding cohomology classes. Our aim is to find a suitable definition for a relative Bennequin number to strengthen these lower bounds and provide lower bounds for all cohomology classes.

We proceed as follows: Given a link $L=L_{1} \cup \cdots \cup L_{r}$ and a class $C=$ $\left(C_{1}, \ldots, C_{r}\right)$ in $H^{1}(M ; \mathbb{Z})$, where $M$ is the link complement, we want to get a lower bound for the Thurston norm which extends the Bennequin inequality.

- First, we change the orientation of $L$ so that the corresponding change of the cohomology basis makes all $C_{i} \geq 0$. 
- Now we transform this reoriented link $L$ into a closed braid, using Vogel's algorithm. This procedure is by no means unique. All our constructions and inequalities from now on will depend on the choice of this braid representative for $L$. So we assume that all $C_{i} \geq 0$ and in addition the link is given as a closed braid.

- In a first theorem we will prove an inequality if all the $C_{i}$ are either 1 or 0 . This corresponds to the question of finding a minimal spanning surface of the sublink of those components of $L$ that correspond to $C_{i}=1$ in the complement of the others.

- The final step is to explicitly construct a link diagram so that the Thurston norm minimizing surface which corresponds to the class $C$ is a minimal spanning surface for that link. Here we can apply the tools that we worked out before to get a lower bound for all $C$ with $C_{i} \geq 0$.

We will call the lower bound the relative Bennequin number. The definition of the relative Bennequin number of subdiagrams of link diagrams is meant to be an extension of the Bennequin number taking into account the crossings of components not in the sublink. Let $\operatorname{cr}\left(L_{i}, L_{j}\right)$ denote the algebraic crossing number between components $L_{i}$ and $L_{j}$ of $L$ and $n_{i}$ the number of Seifert circles in Seifert's algorithm applied to $L_{i}$ alone. We define the relative Bennequin number

$\beta_{t}\left(L_{i}, L\right):=\operatorname{cr}\left(L_{i}, L_{i}\right)-n_{i}+\sum_{j \neq i} \frac{1}{2} \operatorname{cr}\left(L_{i}, L_{j}\right)=\operatorname{cr}\left(L_{i}, L_{i}\right)-n_{i}+\sum_{j \neq i} l k\left(L_{i}, L_{j}\right)$

and define

$$
\beta_{t}\left(L_{i_{1}} \cup \cdots \cup L_{i_{k}}, L\right):=\sum_{j=1}^{k} \beta_{t}\left(L_{i_{j}}, L\right) .
$$

For a closed braid, note that $\beta_{t}(L, L)=\beta_{t}(L)$ since the number of Seifert circles is the sum of the numbers of Seifert circles of the individual components.

We can extend Bennequin's inequality to an inequality between the relative Bennequin number and the Thurston norm.

Theorem 1.4.2 Let $1 \leq i_{1}<i_{2}<\cdots<i_{k} \leq r$ be integers and let $C=$ $\left(C_{1}, \ldots, C_{r}\right)$ where $C_{i}=1$ if $i \in\left\{i_{1}, \ldots i_{k}\right\}$ and $C_{i}=0$ otherwise. Then

$$
\beta_{t}\left(L_{i_{1}} \cup \cdots \cup L_{i_{k}}, L\right) \leq\|C\|_{T} .
$$

Proof Suppose that $S$ is a norm minimizing surface representing $C$. By Lemma 1.2.1 we may assume that $S \cap \partial \mathcal{N}\left(L_{i_{j}}\right)$ is a single simple closed curve 
isotopic to $L_{i_{j}}$ for each $1 \leq j \leq k$ and that $S \cap \partial \mathcal{N}\left(L_{i}\right)$ is no fewer than $\left|\sum_{j=1}^{k} l k\left(L_{i}, L_{i_{j}}\right)\right|$ meridional curves for each $i \notin\left\{i_{1}, \ldots i_{k}\right\}$.

We can fill in each boundary curve of $S$ on $\partial \mathcal{N}\left(L_{i}\right), i \notin\left\{i_{1}, \ldots i_{k}\right\}$ with a meridian disk for $\mathcal{N}\left(L_{i}\right)$ to obtain a Seifert surface $S^{\prime}$ for the link $L_{i_{1}} \cup \cdots \cup L_{i_{k}}$ in $S^{3}$. Then

$$
-\chi\left(S^{\prime}\right) \leq-\chi(S)-\sum_{i \notin\left\{i_{1}, \ldots, i_{k}\right\}}\left|\sum_{j=1}^{k} l k\left(L_{i}, L_{i_{j}}\right)\right| .
$$

Bennequin's inequality tells us that $\beta_{t}\left(L_{i_{1}} \cup \cdots \cup L_{i_{k}}\right) \leq-\chi\left(S^{\prime}\right)$. So

$$
\begin{aligned}
\beta_{t}\left(L_{i_{1}} \cup \cdots \cup L_{i_{k}}, L\right) & =\beta_{t}\left(L_{i_{1}} \cup \cdots \cup L_{i_{k}}\right)+\sum_{i \notin\left\{i_{1}, \ldots, i_{k}\right\}} \sum_{j=1}^{k} l k\left(L_{i}, L_{i_{j}}\right) \\
& \leq \beta_{t}\left(L_{i_{1}} \cup \cdots \cup L_{i_{k}}\right)+\sum_{i \notin\left\{i_{1}, \ldots, i_{k}\right\}}\left|\sum_{j=1}^{k} l k\left(L_{i}, L_{i_{j}}\right)\right| \\
& \leq-\chi\left(S^{\prime}\right)+\sum_{i \notin\left\{i_{1}, \ldots, i_{k}\right\}}\left|\sum_{j=1}^{k} l k\left(L_{i}, L_{i_{j}}\right)\right| \\
& \leq-\chi(S) \\
& \leq \chi_{-}(S) .
\end{aligned}
$$

Remark 1.4.3 In fact, we have proved a somewhat better bound holds. In the proof of Theorem 1.4.2, we showed that the quantity in line (3) is a lower bound for the Thurston norm of the class $C$. This inequality is stronger than the inequality in the statement of the theorem if the total linking of any component $L_{i}$ for $i \notin\left\{i_{1}, \ldots i_{k}\right\}$ with the components $L_{i_{j}}$ is negative.

We would like to make an assertion like Theorem 1.4.2 for any class $C=$ $\left(C_{1}, \ldots, C_{r}\right) \in H^{1}(M ; \mathbb{Z}), C_{i} \geq 0$. To do so, we must associate a relative Bennequin number to classes with coordinates greater than 1 . We will use the closed braid diagram for $L$ to create a new closed braid link diagram $L^{\prime}$ and a subdiagram $L^{\prime \prime}$ so that the pair corresponds to the class $C$. These new link diagrams are derived from the boundary of a Thurston norm minimizing surface $S$ for $C$. More precisely, $L^{\prime}$ is the link constructed by replacing each component $L_{i}$ of $L$ where $C_{i}>0$ with the boundary components of $S$ which lie on the boundary of a regular neighborhood of $L_{i}$. While the complements of $L$ and $L^{\prime}$ are typically not homeomorphic, the surface $S$ exists in both. By passing from $L$ to $L^{\prime}$, we will have arranged for $S$ to be dual to an element in 
$H^{1}\left(S^{3}-\mathcal{N}\left(L^{\prime}\right)\right)$ that is of the form where we can apply the classical Bennequin inequality.

By Lemma 1.2.1 we may assume that a norm minimizing surface $S$ representing $C$ has $S \cap \partial \mathcal{N}\left(L_{i}\right)$ a collection $\gamma_{i}$ of parallel simple closed curves that are essential in $\partial \mathcal{N}\left(L_{i}\right)$ and all oriented in the same direction. Let $\lambda_{i}$ be a longitude for $L_{i}$, that is, a simple closed curve in $\partial \mathcal{N}\left(L_{i}\right)$ such that $\lambda_{i}$ does not link $L_{i}$ and such that the intersection number of $\mu_{i}$ and $\lambda_{i}$ is equal to +1 . We have that $\left[\gamma_{i}\right]=p_{i}\left[\mu_{i}\right]+q_{i}\left[\lambda_{i}\right] \in H_{1}\left(\partial \mathcal{N}\left(L_{i}\right) ; \mathbb{Z}\right)$ where

$$
q_{i}=C_{i} \text { and } p_{i}=-\sum_{j \neq i} C_{j} \cdot l k\left(L_{i}, L_{j}\right) .
$$

We define the two new link diagrams based on the closed braid diagram for $L$. Let $L^{\prime}$ be the link diagram obtained by replacing the link component $L_{i}$ with the curves $\gamma_{i}$ for all $1 \leq i \leq r$ with $C_{i} \neq 0$ as follows. Replace each strand of the component $L_{i}$ by $C_{i}$ parallel strands. Let the new strands replacing the first strand of $L_{i}$ be strands $m_{i}+1, m_{i}+2, \ldots, m_{i}+C_{i}$. At the end of these strands, insert the braid

$$
\left(\sigma_{m_{i}+1} \sigma_{m_{i}+2} \ldots \sigma_{m_{i}+C_{i}-1}\right)^{p_{i}-C_{i} c r\left(L_{i}, L_{i}\right)} .
$$

Here, the $\sigma_{i}$ denote a positive standard generator of the braid group, i.e. the braid has a positive crossing between the $i$-th. and $i+1$-th. strand. We obtain the link diagram $L^{\prime \prime}$ from $L^{\prime}$ by deleting each component $L_{i}$ with $C_{i}=0$. Note that $L^{\prime}$ and $L^{\prime \prime}$ are closed braids and that $L^{\prime \prime}$ is a subdiagram of $L^{\prime}$.

Example 1.4.4 Let $L$ be the closure of the braid $\sigma_{1}^{4}$, which is a 2-component link $L_{1} \cup L_{2}$. Seifert's algorithm gives us a torus with two boundary components. Hence, the Thurston norm at the point $(1,1)$ is less than or equal to 2 .

Indeed, Bennequin's inequality computes as

$$
\beta_{t}(L)=\beta_{t}\left(L_{1} \cup L_{2}\right)=4-2=2 \leq-\chi(S(L)) .
$$

Hence, the Thurston norm is 2 at this point.

The relative Bennequin numbers $\beta_{t}\left(L_{i}, L\right), i=1,2$, are both 1. Again, since the generalized Seifert algorithm gives us a two-punctured disk as a spanning surface of one component in the complement of the other, we get the Thurston norm at $(1,0)$ and $(0,1)$.

Now, look for example at the point $(2,1)$. In the construction above we get $q_{1}=$ $2, q_{2}=1$ and $p_{1}=-2$. Hence, our new closed braid $L^{\prime}=L^{\prime \prime}$ is $\sigma_{2} \sigma_{1}^{2} \sigma_{2}^{2} \sigma_{1}^{2} \sigma_{2} \sigma_{1}^{-2}$ and the relative Bennequin number $\beta_{t}\left(L^{\prime}, L^{\prime}\right)$ equals 3 . 
The Thurston norm is a semi-norm and we get

$$
3=\beta_{t}\left(L^{\prime}, L^{\prime}\right) \leq\|(2,1)\|_{T} \leq\|(1,1)\|_{T}+\|(1,0)\|_{T}=2+1=3 .
$$

Theorem 1.4.5

$$
\beta_{t}\left(L^{\prime \prime}, L^{\prime}\right)=\sum_{i=1}^{r} C_{i} \beta_{t}\left(L_{i}, L\right) .
$$

Proof We first note that $\beta_{t}\left(L^{\prime \prime}, L^{\prime}\right)=\beta_{t}\left(L^{\prime \prime}\right)+1 / 2 c r\left(L^{\prime \prime}, L^{\prime}-L^{\prime \prime}\right)$ where $\operatorname{cr}\left(L^{\prime \prime}, L^{\prime}-L^{\prime \prime}\right)$ is the algebraic crossing number between components in $L^{\prime \prime}$ and components not in $L^{\prime \prime}$.

Crossings in $L^{\prime \prime}$ occur in the following ways:

- For each crossing of $L_{i}$ with itself, there are $C_{i}^{2}$ crossings with the same sign in $L^{\prime \prime}$.

- For each component $L_{i}$, there are $\left(C_{i}-1\right)\left(p_{i}-C_{i} \operatorname{cr}\left(L_{i}, L_{i}\right)\right)$ crossings in $L^{\prime \prime}$ (counted with sign) coming from the braid inserted above.

- For each crossing of $L_{i}$ with $L_{j}$ for $j \neq i$, there are $C_{i} C_{j}$ crossings with the same sign in $L^{\prime \prime}$.

Therefore,

$$
\begin{aligned}
\beta_{t}\left(L^{\prime \prime}\right)= & \sum_{i=1, C_{i} \neq 0}^{r}\left(C_{i}^{2} \operatorname{cr}\left(L_{i}, L_{i}\right)-C_{i}\left(C_{i}-1\right) \operatorname{cr}\left(L_{i}, L_{i}\right)\right. \\
& \left.+p_{i}\left(C_{i}-1\right)+\sum_{j \neq i}\left(C_{i} C_{j} l k\left(L_{i}, L_{j}\right)\right)-C_{i} n_{i}\right) \\
= & \sum_{i=1, C_{i} \neq 0}^{r}\left(C_{i}^{2} \operatorname{cr}\left(L_{i}, L_{i}\right)-C_{i}\left(C_{i}-1\right) \operatorname{cr}\left(L_{i}, L_{i}\right)\right. \\
& \left.-\left(C_{i}-1\right) \sum_{j \neq i}\left(C_{j} l k\left(L_{i}, L_{j}\right)\right)+\sum_{j \neq i}\left(C_{i} C_{j} l k\left(L_{i}, L_{j}\right)\right)-C_{i} n_{i}\right) \\
= & \sum_{i=1, C_{i} \neq 0}^{r}\left(C_{i} \operatorname{cr}\left(L_{i}, L_{i}\right)+\sum_{j \neq i} C_{j} l k\left(L_{i}, L_{j}\right)-C_{i} n_{i}\right) .
\end{aligned}
$$

The sums can be taken over all $j$ in these equations because each relevant term contains a $C_{j}$ factor, eliminating those components in $L^{\prime}-L^{\prime \prime}$. 
Hence,

$$
\begin{aligned}
& \beta_{t}\left(L^{\prime \prime}, L^{\prime}\right)= \\
& =\beta_{t}\left(L^{\prime \prime}\right)+1 / 2 \operatorname{cr}\left(L^{\prime \prime}, L^{\prime}-L^{\prime \prime}\right) \\
& =\sum_{i=1, C_{i} \neq 0}^{r}\left(C_{i} \operatorname{cr}\left(L_{i}, L_{i}\right)+\sum_{j \neq i} C_{j} l k\left(L_{i}, L_{j}\right)-C_{i} n_{i}\right)+1 / 2 c r\left(L^{\prime \prime}, L^{\prime}-L^{\prime \prime}\right) \\
& =\sum_{i=1, C_{i} \neq 0}^{r}\left(C_{i} \operatorname{cr}\left(L_{i}, L_{i}\right)+\sum_{j \neq i} C_{j} l k\left(L_{i}, L_{j}\right)-C_{i} n_{i}+1 / 2 C_{i} \sum_{C_{j}=0} \operatorname{cr}\left(L_{i}, L_{j}\right)\right) \\
& =\sum_{i=1, C_{i} \neq 0}^{r}\left(C_{i} \operatorname{cr}\left(L_{i}, L_{i}\right)+\sum_{j \neq i} C_{j} l k\left(L_{i}, L_{j}\right)-C_{i} n_{i}+C_{i} \sum_{C_{j}=0} l k\left(L_{i}, L_{j}\right)\right) \\
& =\sum_{i=1}^{r}\left(C_{i} \operatorname{cr}\left(L_{i}, L_{i}\right)+C_{i} \sum_{j \neq i} l k\left(L_{i}, L_{j}\right)-C_{i} n_{i}\right) \\
& =\sum_{i=1}^{r} C_{i} \beta_{t}\left(L_{i}, L\right) .
\end{aligned}
$$

The equality in line (5) is true because the second term in line (4) will produce all terms of the form $C_{i} l k\left(L_{i}, L_{j}\right)=C_{i} l k\left(L_{j}, L_{i}\right)$ for all pairs $(i, j)$ with $i \neq j$ and neither $C_{i}$ nor $C_{j}$ equal to zero. The fourth term in line (4) will produce all terms of the form $C_{i} l k\left(L_{i}, L_{j}\right)$ for all pairs $(i, j)$ with $C_{i} \neq 0$ and $C_{j}=0$. Together, they produce all terms of the form $C_{i} l k\left(L_{i}, L_{j}\right)$ for all pairs $(i, j)$ with $i \neq j$ and $C_{i} \neq 0$. The sum in line (5) can be taken over all $i$ because a factor of $C_{i}$ appears in every term, eliminating those with $C_{i}=0$.

With this linearity result on relative Bennequin numbers, we obtain a lower bound on the Thurston norm of any cohomology class $C=\left(C_{1}, \ldots, C_{r}\right)$ in terms of the relative Bennequin numbers of each link component of the closed braid diagram for $L$ reoriented so that all $C_{i} \geq 0$.

Corollary 1.4.6 For any class $C=\left(C_{1}, \ldots, C_{r}\right) \in H^{1}(M ; \mathbb{Z})$,

$$
\beta_{t}\left(L^{\prime \prime}, L^{\prime}\right)=\sum_{i=1}^{r} C_{i} \beta_{t}\left(L_{i}, L\right) \leq\|C\|_{T} .
$$

Proof The pair of link diagrams $L^{\prime}$ and $L^{\prime \prime}$ are defined in terms of a norm minimizing surface $S$ for the class $C$ so that $S$ is also a surface spanning $L^{\prime \prime}$ in the complement of $L^{\prime}$. Therefore, by Theorem 1.4.2, $\beta_{t}\left(L^{\prime \prime}, L^{\prime}\right) \leq \chi_{-}(S)=$ $\|C\|_{T}$. 
As in Remark 1.4.3, our proof of Theorem 1.4.2 provides a better lower bound than the one given in Corollary 1.4.6, although it does not have as succinct an expression. This lower bound is outlined in the Scholium below.

Scholium 1.4.7 For any class $C=\left(C_{1}, \ldots, C_{r}\right) \in H^{1}(M ; \mathbb{Z})$,

$$
\beta_{t}\left(L^{\prime \prime}, L^{\prime}\right) \leq \beta_{t}\left(L^{\prime \prime}\right)+\sum_{i=1, C_{i}=0}^{r}\left|\sum_{j=1}^{r} C_{j} l k\left(L_{i}, L_{j}\right)\right| \leq\|C\|_{T} .
$$

Proof The first inequality is actually an equality if each term in the absolute value signs is non-negative. In this case the statement here is identical to Corollary 1.4.6.

As in the proof of Theorem 1.4.2, a Thurston norm minimizing surface $S$ with no closed components representing $C$ has $S \cap \partial \mathcal{N}\left(L_{i}\right)$ equal to no fewer than $\left|\sum_{j=1}^{r} C_{j} l k\left(L_{i}, L_{j}\right)\right|$ meridian curves for each $i \in\{1, \ldots, r\}$ with $C_{i}=0$. Filling in each of these with a meridian disk produces a spanning surface $S^{\prime \prime}$ for $L^{\prime \prime}$ in $S^{3}$. Then

$$
-\chi\left(S^{\prime \prime}\right) \leq-\chi(S)-\sum_{i=1, C_{i}=0}^{r}\left|\sum_{j=1}^{r} C_{j} l k\left(L_{i}, L_{j}\right)\right| .
$$

From Bennequin's inequality, we know that $\beta_{t}\left(L^{\prime \prime}\right) \leq-\chi\left(S^{\prime \prime}\right)$. Therefore,

$$
\beta_{t}\left(L^{\prime \prime}\right)+\sum_{i=1, C_{i}=0}^{r}\left|\sum_{j=1}^{r} C_{j} l k\left(L_{i}, L_{j}\right)\right| \leq-\chi(S) \leq \chi_{-}(S)=\|C\|_{T} .
$$

\section{The Comparison of the Thurston and the Alexan- der norm}

\subsection{Closed homogeneous braids}

First we need a lemma for arbitrary semi-norms

Lemma 2.1.1 Let $\|$.$\| on \mathbb{R}^{n}$ with basis $e_{i}, i=1, \ldots, n$, be a semi-norm, such that

$$
\left\|\sum_{i=1}^{n} e_{i}\right\|=\sum_{i=1}^{n}\left\|e_{i}\right\|
$$

Then

for all $c_{i} \geq 0$.

$$
\left\|\sum_{i=1}^{n} c_{i} e_{i}\right\|=\sum_{i=1}^{n} c_{i}\left\|e_{i}\right\|
$$

Algebraic 83 Geometric Topology, Volume 1 (2001) 
Proof Assume that the basis $e_{i}$ is chosen so that $\left\|e_{i}\right\| \neq 0$ for $i \leq n_{1}$ and $\left\|e_{i}\right\|=0$ for $n_{1}<i \leq n$. The unit ball $B$ for $\|$.$\| is convex. Therefore B$ is an intersection of half-spaces. If a point $v \in \mathbb{R}^{n}$ has norm $\|v\|=1$ then there exists an $(n-1)$-dimensional affine space $\mathcal{A}$ through $v$ such that $B$ is contained in the closure of one of two halfspaces defined by $\mathcal{A}$. Let $v_{i}$ be the point $e_{i} /\left\|e_{i}\right\|$ for $i \leq n_{1}$. Choose $v=\left(\sum e_{i}\right) /\left(\sum\left\|e_{i}\right\|\right)$, hence $\|v\|=1$. The only $(n-1)$ dimensional affine space $\mathcal{A}$ that contains $v$, and has a closed halfspace that contains all $v_{i}, i \leq n_{i}$, and all $m_{i} e_{i}, i>n_{1}$, is the $\left(n_{1}-1\right)$-dimensional affine space containing the $v_{i}$ 's times $R^{n-n_{1}}$.

For all $w \in \mathcal{A} \cap \mathbb{R}_{\geq 0}^{n}$ it follows $\|w\|=1$.

We get:

Theorem 2.1.2 Let $\mathfrak{b}$ be a braid so that Seifert's algorithm gives a minimal spanning surface for its closure $\hat{\mathfrak{b}}$ and let $M=S^{3}-\mathcal{N}(\hat{\mathfrak{b}})$ be the complement. Assume furthermore that $\hat{\mathfrak{b}}$ has no unlinked unknotted components. Number the components of $\hat{\mathfrak{b}}$ and take as a basis for $H^{1}(M ; \mathbb{Z})$ the one induced by the meridians as described in Section 1.

The Thurston norm is additive for all $\left(C_{1}, \ldots, C_{r}\right) \in H^{1}(M ; \mathbb{Z})$ with $C_{j} \geq$ 0 . Its value on the points $\left(0, \ldots, 0, C_{j}=1,0, \ldots, 0\right)$ equals minus the Euler characteristic of the spanning surface for the $j$-th component pierced by the other components that we get as described in Section 1.1.

Proof First we prove the theorem for the case that all $C_{i}$ are equal to either 0 or 1 . By assumption the Thurston norm at the point $(1, \ldots, 1)$ equals

$$
\|(1, \ldots, 1)\|_{T}=l-n
$$

where $l$ is the word length of the braid and $n$ the number of strands.

Choose a component $L_{j}$ with $n_{j}$ strands of the link. Construct a surface that spans this component as in the generalization of Seifert's algorithm explained in Section 1.1. That is, if another component undercrosses an $\operatorname{arc}$ of $L_{j}$, we get a piercing of the surface. Let $u_{j}$ be the number of these piercings and $l_{j}$ be the number of self-crossings of the component $L_{j}$. The so constructed surface has Euler characteristic

$$
n_{j}-u_{j}-l_{j}
$$

and thus, the Thurston norm at the point $\left(0, \ldots, 0, C_{j}=1,0, \ldots 0\right)$ is less than or equal to $u_{j}+l_{j}-n_{j}$. By definition $n=\sum n_{j}$ and $l=\sum\left(l_{j}+u_{j}\right)$. 
Since the Thurston norm is a semi-norm, we have

$$
\begin{aligned}
l-n=\|(1, \ldots, 1)\|_{T} & \leq \sum_{j}\left\|\left(0, \ldots, 0, C_{j}=1,0, \ldots, 0\right)\right\|_{T} \\
& \leq \sum_{j}\left(u_{j}+l_{j}-n_{j}\right)=l-n .
\end{aligned}
$$

Thus, the Thurston norm of $\left(0, \ldots, 0, C_{j}=1,0, \ldots, 0\right)$ equals $l_{j}+u_{j}-n_{j}$.

Lemma 2.1.1 gives us the additivity for all other points $\left(C_{1}, \ldots, C_{r}\right)$ with $C_{i} \geq$ 0 .

Proposition 2.1.3 Assume the conditions for the Thurston norm as in Theorem 2.1.2 are fulfilled. Assume furthermore, that the Alexander norm at the point $(1, \ldots, 1) \in H^{1}(M ; \mathbb{Z})$ equals the Thurston norm.

Then the Alexander norm equals the Thurston norm for all points $\left(C_{1}, \ldots, C_{r}\right)$ with $C_{i} \geq 0$.

Proof If the closed braid is a knot, then the theorem is immediate. Hence, we assume that $\hat{\mathfrak{b}}$ has at least two components. Since the Alexander norm is bounded from above by the Thurston norm, it follows that for $C^{(j)}=$ $\left(0, \ldots, 0, C_{j}=1,0, \ldots, 0\right)$,

$$
\left\|\left(0, \ldots, 0, C_{j}=1,0, \ldots, 0\right)\right\|_{A}=l_{j}+u_{j}-n_{j}=\left\|\left(0, \ldots, 0, C_{j}=1,0, \ldots, 0\right)\right\|_{T} .
$$

Now, Lemma 2.1.1 shows that the Alexander norm is also additive on all points $C=\left(C_{1}, \ldots, C_{r}\right)$ with $C_{i} \geq 0$. Therefore,

$$
\|C\|_{A}=\sum_{j=1}^{r} C_{j}\left\|C^{(j)}\right\|_{A}=\sum_{j=1}^{r} C_{j}\left\|C^{(j)}\right\|_{T}=\|C\|_{T} .
$$

An important class of links are closures of homogeneous braids, i.e. the braid contains every standard generator $\sigma_{i}$ of the braid group at least once and always with the same exponent sign.

Corollary 2.1.4 For closed homogeneous braids the Alexander norm equals the Thurston norm for all points $\left(C_{1}, \ldots, C_{r}\right) \in H^{1}(M ; \mathbb{Z})$ with $C_{i} \geq 0$.

Proof By a theorem of Stallings [Sta78] these links are fibred and the fibre surface is the one that one gets by the application of Seifert's algorithm to the closed braid.

Hence, the Alexander norm equals the Thurston norm at the point $(1, \ldots, 1)$ (e.g. [McM99]) and the claim follows by Proposition 2.1.3. 


\section{The Bennequin number and the Thurston norm}

\subsection{The Bennequin number and the band generators}

Recently, a new presentation for the braid groups began to receive attention: a presentation in terms of the band generators, defined by Birman, Ko and Lee [BKL98]. Let

$$
a_{i, j}:=\sigma_{i}^{-1} \cdots \sigma_{j-2}^{-1} \sigma_{j-1} \sigma_{j-2} \cdots \sigma_{i},
$$

where $\sigma_{i}$ are the standard generators (see Figure 4). In [BKL98] a solution to the word problem and conjugacy problem with respect to these band generators is given.

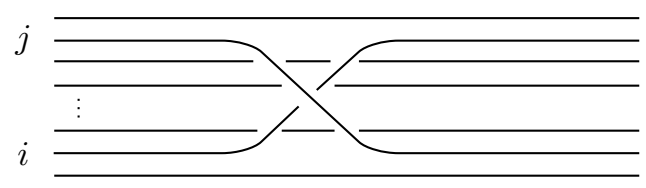

Figure 4: The band generator $a_{i, j}$

For a closed braid given in terms of the band generators, we get a spanning surface by a generalized Seifert algorithm: the number of Seifert disks equals the number of strands, and if $a_{i, j}$ or $a_{i, j}^{-1}$ occurs in a word, then we connect the $i$-th and $j$-th disk by a band. Thus, the Euler characteristic of this spanning surface is $n-l$, where $l$ is the word length in the band generators.

For $\mathfrak{b} \in B_{n}$ a braid, let $\operatorname{pos}_{b}(\mathfrak{b})$ be the positive exponent sum in the band generators and $\operatorname{neg}_{b}(\mathfrak{b})$ be the negative exponent sum. Bennequin's inequality gives us

$$
\operatorname{pos}_{b}(\mathfrak{b})-\operatorname{neg}_{b}(\mathfrak{b})-n \leq-\chi(S(\hat{\mathfrak{b}}))
$$

for any spanning surface of the closed braid $\hat{\mathfrak{b}}$.

A braid $\mathfrak{b}$ is band positive if $\operatorname{neg}_{b}(\mathfrak{b})=0$. (This is a different terminology than in the work of Rudolph (see e.g. [Rud99] and the references therein). There, band positive braids are a special type of quasi-positive braids.)

Bennequin's inequality gives the following proposition which is an extension of the main result of Ko and Lee in [KL97], where it is proved, with different methods, if the number of strands equals 4 .

Proposition 3.1.1 Let $\mathfrak{b}$ be a band positive braid in $B_{n}$ of length $l$. Then the Euler characteristic of a minimal spanning surface satisfies: $-\chi(S(\hat{\mathfrak{b}}))=l-n$. 
A special class of band positive braids is particularly well-suited for computing the Thurston norm at the cohomology classes $C=\left(C_{1}, \ldots, C_{r}\right)$ with $C_{i} \geq 0$. This class consists of band positive braids with only bands of the form $a_{i, i+1}$ or $a_{i, j}$ such that the $i$ th and $j$ th strands are parts of the same link component in the closed braid. This class of braids has the property that every sublink is also band positive.

Proposition 3.1.2 For a closed braid in the class described above with no unlinked, unknotted components, the relative Bennequin inequality in Corollary 1.4.6 is an equality for every cohomology class $C=\left(C_{1}, \ldots, C_{r}\right)$ with $C_{i} \geq 0$.

Proof First, on the points $C^{(i)}=\left(0, \ldots, 0, C_{i}=1,0, \ldots, 0\right)$ the relative Bennequin inequality is $\beta_{t}\left(L_{i}, L\right) \leq\left\|C^{(i)}\right\|_{T}$. On the other hand, a generalized Seifert band algorithm similar to the generalized Seifert algorithm for the standard generators described in Section 1.1 produces a surface $S_{i}$ with $\chi_{-}\left(S_{i}\right)=-\chi\left(S_{i}\right)=\beta_{t}\left(L_{i}, L\right)$.

Hence, we know that the relative Bennequin inequality is an equality for all points $C^{(i)}$ and in addition for $(1, \ldots, 1)$. Thus, by Lemma 2.1.1, the Thurston norm is additive on all points $\left(C_{1}, \ldots, C_{r}\right)$ with $C_{i} \geq 0$. Since we know by Theorem 1.4.5 that the relative Bennequin number is also additive on these points, we are done.

Note that, in contrast, the estimate on the Alexander norm coming from the Thurston norm for this class of closed braids is in general not sharp. In [KL97] an example of a knot represented by a closed band positive braid is given, where the Thurston norm equals 3, while the Alexander norm is 2 .

Using this example one can easily find more examples of links with more components. The computation was made with the help of the program [MH96] based on a description of the multivariable Alexander polynomial due to Morton [Mor99].

The two component link represented by the closure of the 5 -braid

$$
a_{4,5}^{2} a_{2,4}^{2} a_{1,3} a_{3,4} a_{2,4} a_{1,3}^{2}
$$

has Alexander polynomial $2-3 t_{1}+2 t_{1}^{2}$ (the second variable vanishes), hence the Alexander norm at $(1,1)$ is 2 . The Thurston norm equals 4 , by Proposition 3.1.1. 


\section{The HOMFLY polynomial and a conjecture of Morton}

In the last section we deal with another invariant for links which is conjectured to also give a lower bound for the Thurston norm. It comes from the HOMFLY polynomial. Shortly after the HOMFLY polynomial was defined, Hugh Morton [Mor86] and independently Franks and Williams [FW87] realized that its lowest degree in one of the variables can be estimated by the Bennequin number (see e.g. [Lic88] for a still excellent account to the, by now, classical knot polynomials).

We briefly recall the relevant results. On braids let a two-variable (Laurent-) polynomial $P(\mathfrak{b})(v, z)$ be defined by:

$$
\begin{aligned}
P\left(\mathfrak{b} \sigma_{i}\right)-P\left(\mathfrak{b} \sigma_{i}^{-1}\right) & =z P(\mathfrak{b}), \quad 1 \leq i \leq n-1 \\
P\left(\mathfrak{b} \sigma_{n}\right) & =P(\mathfrak{b}) \\
P\left(\mathfrak{b} \sigma_{n}^{-1}\right) & =v^{2} P(\mathfrak{b}) \\
P\left(\mathrm{id}_{n}\right) & =\left(\left(1-v^{2}\right) / z\right)^{n}
\end{aligned}
$$

Here $\mathfrak{b}$ is assumed to be a braid in $B_{n}$, thus $\sigma_{n}$ does not occur in $\mathfrak{b}$, and $\operatorname{id}_{n}$ is the identity in $B_{n}$. Furthermore, the polynomial is, by definition, invariant under braid isotopy, i.e. relations in $B_{n}$, and conjugation. From the definition it follows that if the braid has the form $\operatorname{id}_{n} \mathfrak{b}$ with $\mathfrak{b} \in B_{n-1}$ then

$$
P\left(\operatorname{id}_{n} \mathfrak{b}\right)=\left(\left(1-v^{2}\right) / z\right) P(\mathfrak{b}) .
$$

It is easy to see, that

$$
v^{\beta_{t}(\mathfrak{b})} P(\mathfrak{b})=\frac{\left(v^{-1}-v\right)}{z} \operatorname{HOMFLY}(\hat{\mathfrak{b}})
$$

where $\operatorname{HOMFLY}(\hat{\mathfrak{b}})$ is the HOMFLY polynomial of the closure $\hat{\mathfrak{b}}$ of $\mathfrak{b}$ and $\beta_{t}(\mathfrak{b})$ is again the Bennequin number of the braid $\mathfrak{b}$, i.e. $\operatorname{pos}(\mathfrak{b})-\operatorname{neg}(\mathfrak{b})-n$. This gives that $P$, assuming the definitions are sufficient, is unique and well-defined. To see that the definition is sufficient to compute $P$, notice that

$$
\begin{aligned}
P\left(\mathfrak{b} \sigma_{i}^{2}\right) & =P(\mathfrak{b})+z P\left(\mathfrak{b} \sigma_{i}\right) \\
P\left(\mathfrak{b} \sigma_{i}^{-1}\right) & =P\left(\mathfrak{b} \sigma_{i}\right)-z P(\mathfrak{b}) .
\end{aligned}
$$

With (12) one can assume that it is sufficient to compute $P$ for all positive braids. Relations (11), (7), (8) and (9) are now sufficient to compute $P$ without having to increase the word length. 
The specialization $v:=1$ in

$$
z P(v, z)(\mathfrak{b}) /\left(1-v^{2}\right)
$$

is the (one variable) Alexander polynomial of the closed braid $\hat{\mathfrak{b}}$ in its Conway form.

From the definition of $P$ and its computation it follows immediately that $P$ is a polynomial in $v$ rather than a Laurent polynomial. Therefore, if $e(\hat{\mathfrak{b}})$ is the minimum degree of $v$ in $\operatorname{HOMFLY}(\hat{\mathfrak{b}})$ we have

Theorem 4.0.3 (Morton [Mor86], Franks-Williams [FW87]) Let $\hat{\mathfrak{b}}$ be the closure of a braid $\mathfrak{b}$ and $e(\hat{\mathfrak{b}})$ be the minimum degree of $v$ in the HOMFLY polynomial of $\hat{\mathfrak{b}}$. Then

$$
\beta_{t}(\mathfrak{b})+1 \leq e(\hat{\mathfrak{b}})
$$

This theorem turned out to be a powerful tool in Legendrian and transversal knot theory, as an upper bound for the Bennequin number (see [FT97, Tab97, CG97]).

As a corollary we get:

Corollary 4.0.4 If, for a braid $\mathfrak{b}$, the polynomial $P(\mathfrak{b})(0, z) \neq 0$ then the Bennequin number of $\mathfrak{b}$ is maximal among the Bennequin numbers of all representatives of the closure $\hat{\mathfrak{b}}$ of $\mathfrak{b}$.

In [Mor86] Hugh Morton made the intriguing conjecture:

Conjecture 4.0.5 (Morton) For a link $L$ let $e(L)$ be the lowest degree of the HOMFLY polynomial in the framing variable $v$. Then

$$
e(L) \leq-\chi(S(L))+1
$$

for every Seifert surface $S(L)$ of $L$.

This conjecture and Theorem 4.0.3 again would imply the Bennequin inequality.

\subsection{Homogeneous braids}

We discovered that the corollary motivating the following proposition can be seen immediately from results proven by Cromwell in [Cro89]. However, since our approach is braid theoretical rather than based on knot diagrams, we include a proof. 
Proposition 4.1.1 Let $\mathfrak{b}$ be a homogeneous braid in $B_{n}$ such that every generator occurs at least once. Let $n_{n}$ be the number of generators $\sigma_{i}$ that occur with negative exponents and $n_{p}$ be the number of generators that occur with positive exponents. Thus, $n=n_{n}+n_{p}+1$. Then the polynomial $P(\mathfrak{b})(v, z)$ contains a unique highest term in $z$ :

$$
(-1)^{\operatorname{neg}(\mathfrak{b})-n_{n}}\left(1-v^{2}\right) v^{2 n_{n}} z^{|\mathfrak{b}|-n}
$$

where $|\mathfrak{b}|$ is the word length of $\mathfrak{b}$.

Proof We will apply the following elementary equations:

$$
\begin{aligned}
P\left(\mathfrak{b} \sigma_{i}^{2}\right)= & P(\mathfrak{b})+z P\left(\mathfrak{b} \sigma_{i}\right) \\
P\left(\mathfrak{b} \sigma_{i}^{-2}\right)= & P(\mathfrak{b})-z P\left(\mathfrak{b} \sigma_{i}^{-1}\right) \\
P\left(\mathfrak{b} \sigma_{n}^{-1} \sigma_{n-1} \sigma_{n}^{-1}\right)= & P\left(\mathfrak{b} \sigma_{n-1} \sigma_{n}^{-1} \sigma_{n-1}\right)-z P\left(\mathfrak{b} \sigma_{n}^{-1} \sigma_{n-1}\right) \\
& -z P\left(\mathfrak{b} \sigma_{n-1} \sigma_{n}^{-1}\right) \\
P\left(\mathfrak{b} \sigma_{n} \sigma_{n-1}^{-1} \sigma_{n}\right)= & P\left(\mathfrak{b} \sigma_{n-1}^{-1} \sigma_{n} \sigma_{n-1}^{-1}\right)+z P\left(\mathfrak{b} \sigma_{n-1}^{-1} \sigma_{n}\right)+z P\left(\mathfrak{b} \sigma_{n} \sigma_{n-1}^{-1}\right)
\end{aligned}
$$

If the proposition is proven for the terms on the right hand side, then it is also true for the ones on the left hand side.

First, the proposition is true in $B_{2}$, i.e. for braids $\sigma_{1}^{k}$ or $\sigma_{1}^{-k}$ by induction on $k$. For the representative of the unknot $\sigma_{n-1}^{\epsilon_{n-1}} \cdots \sigma_{1}^{\epsilon_{1}}, \epsilon_{i}= \pm 1$, it is also true.

It is enough to prove the claim, that every homogeneous braid in $B_{n}$ can be brought with the transformations (13)-(16) and the braid relations $\sigma_{i+1} \sigma_{i} \sigma_{i+1}=$ $\sigma_{i} \sigma_{i+1} \sigma_{i}$ into one in which $\sigma_{n-1}$ occurs at most once, modulo words of lesser length.

For this let the claim already be proven for $B_{n-1}$. Thus, we can assume that a braid $\mathfrak{b}:=w_{1} \sigma_{n-1}^{\epsilon_{n-1}} w_{2} \sigma_{n-1}^{\epsilon_{n-1}}, w_{2} \in B_{n-1}$, modulo terms of lower word length, has in $w_{2}$ at most one term $\sigma_{n-2}^{\epsilon_{n-2}}$. If $w_{2}$ is empty then with (13) or (14) we can reduce the word length.

Otherwise, since $\sigma_{i}$ commutes with $\sigma_{n-1}$ for $i<n-2$, we can apply (15),(16) or the braid relations to bring $\mathfrak{b}$ into a form in which $\sigma_{n-1}$ occurs less and which is still homogeneous.

Corollary 4.1.2 [Cro89] Morton's conjecture is true for closed homogeneous braids.

Proof By Proposition 4.1.1 and with the notations there, we know that the minimum degree $e(\hat{\mathfrak{b}})$ of the HOMFLY polynomial is at most $\beta_{t}(\mathfrak{b})+1+2 n_{n}$. 
Since

we have

$$
\begin{aligned}
\beta_{t}(\mathfrak{b}) & =\operatorname{pos}(\mathfrak{b})-\operatorname{neg}(\mathfrak{b})-n_{n}-n_{p}-1 \quad \text { and } \\
-\chi(\hat{\mathfrak{b}}) & =\operatorname{pos}(\mathfrak{b})+\operatorname{neg}(\mathfrak{b})-n_{n}-n_{p}-1
\end{aligned}
$$

$$
\begin{aligned}
e(\hat{\mathfrak{b}}) & \leq \beta_{t}(\mathfrak{b})+1+2 n_{n} \\
& =-\chi(\hat{\mathfrak{b}})-2 \operatorname{neg}(\mathfrak{b})+1+2 n_{n} \\
& \leq-\chi(\hat{\mathfrak{b}})+1 .
\end{aligned}
$$

The last equation follows, since $n_{n} \leq \operatorname{neg}(\mathfrak{b})$.

\subsection{Closed 3-braids}

The aim of this section is to prove Morton's conjecture for closed 3-braids. The idea that lies behind the proof is quite general, though:

(1) We assume the link to be given as a closed braid $\mathfrak{b}$ on $n$ strands in the band generators, such that the band Seifert algorithm (see Section 3.1) yields a minimal spanning surface $S$.

Thus, we get for the Euler characteristic:

$$
-\chi(\hat{\mathfrak{b}})=-\chi(S)=\operatorname{wordlength}(\mathfrak{b})-n=\operatorname{pos}(\mathfrak{b})+\operatorname{neg}(\mathfrak{b})-n,
$$

where, again, $\operatorname{pos}(\mathfrak{b})(\operatorname{neg}(\mathfrak{b})$, respectively) is the number of positive (negative) band generators in the word $\mathfrak{b}$.

(2) The Bennequin number computes as

$$
\beta_{t}(\mathfrak{b})=\operatorname{pos}(\mathfrak{b})-\operatorname{neg}(\mathfrak{b})-n .
$$

(3) Morton's conjecture now states that

$$
e_{P}(\mathfrak{b})+\beta_{t}(\mathfrak{b}) \leq-\chi(\hat{\mathfrak{b}})
$$

where $e_{P}(\mathfrak{b})$ is the lowest degree of the polynomial $P(\mathfrak{b})(v, z)$ in the variable $v$.

Thus, using Equations (17) and (18) we can reformulate Equation (19) to get:

$$
\begin{aligned}
e_{P}(\mathfrak{b})+\operatorname{pos}(\mathfrak{b})-\operatorname{neg}(\mathfrak{b})-n & \leq \operatorname{pos}(\mathfrak{b})+\operatorname{neg}(\mathfrak{b})-n \\
\Longleftrightarrow e_{P}(\mathfrak{b}) & \leq 2 \operatorname{neg}(\mathfrak{b}) .
\end{aligned}
$$

Since $e_{P}(\mathfrak{b}) \leq 2(n-1)$ by the definition of $P(\mathfrak{b})(v, z)$, it remains to check Morton's conjecture for all words with

$$
\operatorname{neg}(\mathfrak{b})<n-1 \text {. }
$$

In the special case $n=3$ we can prove with this idea: 
Theorem 4.2.1 Morton's conjecture is true for any closed 3-braid.

Proof Let again $a_{1,2}:=\sigma_{1}, a_{2,3}:=\sigma_{2}$ and $a_{1,3}:=\sigma_{1}^{-1} \sigma_{2} \sigma_{1}$. With these band generators, the braid group $B_{3}$ has a presentation

$$
B_{3} \cong\left\langle a_{1,2}, a_{2,3}, a_{1,3} \mid a_{1,2} a_{1,3}=a_{2,3} a_{1,2}=a_{1,3} a_{2,3}\right\rangle .
$$

It is known by work of Birman and Menasco [BM93] and of Xu [Xu92] that a Seifert surface with maximal Euler characteristic is realized by a shortest word in these three generators. This means, if a closed 3-braid $\hat{\mathfrak{b}}$ is represented by a shortest word of length $l$ then a minimal spanning surface $S(\hat{\mathfrak{b}})$ has $-\chi(S(\hat{\mathfrak{b}}))=$ $l-3$. It is the surface that we get by the band Seifert algorithm described in Section 3.1.

Now Morton's conjecture claims that the Bennequin number of $\mathfrak{b}$ and $-\chi$ of a minimal spanning surface for $\hat{\mathfrak{b}}$ differ at least by the minimum degree of $v$ in $P(\mathfrak{b})(v, z)$.

By the computation of $P$ it is clear that for a 3-braid the only possible powers of $v$ in the monomials of $P$ are $0,2,4$, and 6. Furthermore, $P$ is divisible by $\left(\left(1-v^{2}\right) / z\right)$ and thus the minimum degree of $v$ in $P(v, z)$ can be at most 4 .

Now, let $\mathfrak{b}$ be a 3 -braid, which is of minimal length, with exponent $\operatorname{sum} \operatorname{neg}(\mathfrak{b})$ over all negative exponents and $\operatorname{pos}(\mathfrak{b})$ over all positive exponents. Thus, the difference between the Bennequin number and $-\chi$ is $2 \operatorname{neg}(\mathfrak{b})$. So, we only have to check Morton's conjecture for braids $\mathfrak{b}$ with neg $(\mathfrak{b}) \leq 1$.

First assume, that $\mathfrak{b}$ is a positive word in the generators. Our claim is that the highest degree in $z$ is $l-3$ where $l$ is the word length. Moreover, we claim that coefficient of $z^{l-3}$ which is a polynomial in $v$ has constant term 1 .

If the word length is one then, as one might easily check, $P(\mathfrak{b})=\left(\left(1-v^{2}\right) / z\right)^{2}$. If the word length is two, the verification of the claim is an easy case-by-case check as well.

Assume that the word length is at least 3. If a square of a generator occurs, then for any of the generators $a=a_{i, j}$ we have

$$
P\left(\mathfrak{b} a^{2}\right)=P(\mathfrak{b})+z P(\mathfrak{b} a)
$$

and the claim holds by induction. Since we have the relations (20) we can thus assume that any subword of length three is of the form $a_{1,2} a_{2,3} a_{1,3}$ or a cyclic permutation of it. 
Now

$$
\begin{aligned}
P\left(\mathfrak{b} a_{1,2} a_{2,3} a_{1,3}\right) & =P\left(\mathfrak{b} a_{1,2}^{-1} a_{2,3} a_{1,3}\right)+z P\left(\mathfrak{b} a_{2,3} a_{1,3}\right) \\
& =P\left(\mathfrak{b} a_{1,2}^{-1} a_{2,3}^{-1} a_{1,3}\right)+z P\left(\mathfrak{b} a_{1,2}^{-1} a_{1,3}\right)+z P\left(\mathfrak{b} a_{2,3} a_{1,3}\right) \\
& =P\left(\mathfrak{b} a_{2,3}^{-1}\right)+z P\left(\mathfrak{b} a_{1,2} a_{1,3}\right)-z^{2} P\left(\mathfrak{b} a_{1,3}\right)+z P\left(\mathfrak{b} a_{2,3} a_{1,3}\right) .
\end{aligned}
$$

By the induction hypothesis the claim follows. By cyclic permutation the claim also follows for the other two words of length three.

Now assume, that neg( $(\mathfrak{b})=1$. So, the closed braid can be assumed as $a^{-1} \pi$ where $\pi$ is a positive braid, with respect to the band generators, and $a$ is one of these generators. Again the braid is assumed to be of minimal length.

Let $S_{+}, S_{-}$and $S_{0}$ be minimal spanning surfaces for the closed braids $\mathfrak{b} a$, $\mathfrak{b} a^{-1}$ and $\mathfrak{b}$, where $a$ is a generator of $B_{3}$. Essential to our arguments is a theorem of Scharlemann and Thompson [ST89]. Two of the three values

$$
-\chi\left(S_{+}\right),-\chi\left(S_{-}\right) \quad \text { and } \quad-\chi\left(S_{0}\right)+1
$$

are the same and the third one is not bigger than the other two. We use the theorem in the following way: In each application of the skein relation (6) one of the three terms in (22) is strictly less than one of the others. Hence, the third one has to be equal to the larger one.

Our claim is that the highest degree in $z$ is $l-3$, where $l$ is the minimal word length; this equals $-\chi$ of a minimal spanning surface for the braid. Its coefficient has a non-trivial term in $v^{2}$. So, Morton's conjecture follows from this.

If the braid $a^{-1} \pi$ contains a square in $\pi$, then we can reduce the braid with the help of (21). Otherwise, we can assume that every positive subword of length three is of the form $a_{1,2} a_{2,3} a_{1,3}$ or a cyclic permutation of it.

If the word length is one then $P(v, z)=v^{2}\left(\left(1-v^{2}\right) / z\right)^{2}$. If the word has length two then an easy computation shows that $P(v, z)=\left(v^{2}-v^{4}\right) / z$.

Now assume, that the word starts with $a_{1,2}^{-1} a_{2,3} a_{1,3}$ We have for some positive word $\pi$

$$
\begin{aligned}
P\left(a_{1,2}^{-1} a_{2,3} a_{1,3} \pi\right) & =P\left(a_{1,2}^{-1} a_{2,3}^{-1} a_{1,3} \pi\right)+z P\left(a_{1,2}^{-1} a_{1,3} \pi\right) \\
& =P\left(a_{2,3}^{-1} \pi\right)+z P\left(a_{1,2}^{-1} a_{1,3} \pi\right) .
\end{aligned}
$$

By the result of Scharlemann and Thompson the word in the second term in the last equation has to have minimal braid length and thus the claim follows by induction. With the same argument the claim follows if the word starts with $a_{2,3}^{-1} a_{1,3} a_{12}$ or $a_{1,3}^{-1} a_{1,2} a_{2,3}$. 
If the word starts with $a_{1,2}^{-1} a_{1,3} a_{1,2}$ it has to be of length at least 4 , otherwise it wouldn't be minimal. Since we already assumed that it is square-free, it has to start with $a_{1,2}^{-1} a_{1,3} a_{1,2} a_{2,3}$, thus

$$
\begin{aligned}
P & \left(a_{1,2}^{-1} a_{1,3} a_{1,2} a_{2,3} \pi\right)= \\
& =P\left(a_{1,2}^{-1} a_{1,3}^{-1} a_{1,2} a_{2,3} \pi\right)+z P\left(a_{2,3} \pi\right) \\
& =P\left(a_{1,2}^{-1} a_{1,3}^{-1} a_{1,2}^{-1} a_{2,3} \pi\right)+z P\left(a_{1,2}^{-1} a_{1,3}^{-1} a_{2,3} \pi\right)+z P\left(a_{2,3} \pi\right) \\
& =P\left(a_{1,2}^{-2} \pi\right)+z P\left(a_{1,2}^{-1} a_{1,3}^{-1} a_{2,3} \pi\right)+z P\left(a_{2,3} \pi\right) \\
& =P(\pi)+z P\left(a_{1,2}^{-1} \pi\right)+z P\left(a_{1,2}^{-1} a_{1,3} a_{2,3} \pi\right)-z^{2} P\left(a_{1,2}^{-1} a_{2,3} \pi\right)+z P\left(a_{2,3} \pi\right) \\
& =P(\pi)+z P\left(a_{1,2}^{-1} \pi\right)+z P\left(a_{1,3} \pi\right)-z^{2} P\left(a_{1,2}^{-1} a_{2,3} \pi\right)+z P\left(a_{2,3} \pi\right) .
\end{aligned}
$$

By induction and using the theorem of Scharlemann and Thompson, it follows now that the highest term is coming from $z^{2} P\left(a_{1,2}^{-1} a_{2,3} \pi\right)$.

If $a_{2,3}^{-1} a_{1,2} a_{2,3}$ or $a_{1,3}^{-1} a_{2,3} a_{1,3}$ are the initial subwords then the claim follows in the same way. This completes the proof.

\subsection{The difference between the Bennequin number and the Thurston norm}

For a link $L$ let $\max \beta_{t}(L)$ be the maximal Bennequin number among all representatives of $L$ and its mirror image and let $S(L)$ be a minimal spanning surface for $L$. (For definitions see Section 1.4) We give a short argument for the recent result of Kanda [Kan98]:

Proposition 4.3.1 [Kan98] The difference between $\max \beta_{t}(L)$ and $-\chi(S(L))$ can be arbitrarily large.

Proof For $k$ odd let $\mathfrak{b}$ be $\sigma_{2}^{-k} \sigma_{1}^{k}$. Since $\mathfrak{b}$ is homogeneous the closure of $\mathfrak{b}$ has as maximal Euler characteristic over all spanning surfaces $3-2 k$. Obviously $\hat{\mathfrak{b}}$ is equivalent to its mirror image.

It follows immediately from the last section that the difference between the maximal Bennequin number among any representative of $\hat{\mathfrak{b}}$ and the Bennequin number of the special $\mathfrak{b}$ is at most 4 . Thus, $\max \beta_{t}(L)$ is less than or equal to $-3+4=1$. The claim follows.

It can actually be easily shown that if the braid has the form $\sigma_{2}^{-l} \sigma_{1}^{k}$ with positive $k$ and $l \geq 2$ then the maximal possible Bennequin number among all representatives of the closure of the braid is $-l+k-3$. For this we show that 
the polynomial $P(0, z)\left(\sigma_{2}^{-l} \sigma_{1}^{k}\right)$ does not vanish for $l \geq 2$. Thus, with Corollary 4.0.4 we get the desired result.

We already know by the proof of Theorem 4.2.1 that the claim is true for the closed 2-braid $\sigma_{1}^{k}$. By Equation (10) it follows that

$$
P(0, z)\left(\operatorname{id}_{3} \sigma_{1}^{k}\right)=1 / z P(0, z)\left(\sigma_{1}^{k}\right) .
$$

Hence, using the defining relations for $P$ we get

$$
\begin{aligned}
P(0, z)\left(\sigma_{2}^{-1} \sigma_{1}^{k}\right) & =0 \\
P(0, z)\left(\sigma_{2}^{-2} \sigma_{1}^{k}\right) & =P(0, z)\left(\operatorname{id}_{3} \sigma_{1}^{k}\right)-z P(0, z)\left(\sigma_{2}^{-1} \sigma_{1}^{k}\right) \\
& =1 / z P(0, z)\left(\sigma_{1}^{k}\right) \\
P(0, z)\left(\sigma_{2}^{-l} \sigma_{1}^{k}\right) & =P(0, z)\left(\sigma_{2}^{-l+2} \sigma_{1}^{k}\right)-z P(0, z)\left(\sigma_{2}^{-l+1} \sigma_{1}^{k}\right) .
\end{aligned}
$$

By induction we can conclude that every $P(0, z)\left(\sigma_{2}^{-l} \sigma_{1}^{k}\right)$ is a polynomial

$$
f(z) P(0, z)\left(\sigma_{1}^{k}\right) \text {. }
$$

For $l \geq 3$ odd, all coefficients in $f(z)$ are negative, and for $l$ even, all coefficients are positive. Thus, cancellation cannot occur and $f(z)$ is nontrivial for all $l \geq 2$.

Note, that in contrast to this result, the difference between the maximal Bennequin number and the minimal degree of the HOMFLY polynomial in the framing invariant $v$ can be at most $2 n-2$ where $n$ is the number of strands of the representative.

\section{References}

[Ben83] D. Bennequin, Entrelacement et équations de Pfaff, Astérisque 107 - 108 (1983), 83-161 (French).

[BKL98] J. S. Birman, K. H. Ko, and S. J. Lee, A new approach to the word and conjugacy problems in the braid groups, Adv. Math. 139 (1998), no. 2, 322-353.

[BM91] J. S. Birman and W. W. Menasco, Studying links via closed braids. II. On a theorem of Bennequin, Topology Appl. 40 (1991), no. 1, 71-82.

[BM93] J. S. Birman and W. W. Menasco, Studying links via closed braids III. Classifying links which are closed 3-braids, Pacific J. Math. 161 (1993), no. 1, 25-113.

[BW99] J. S. Birman and N. C. Wrinkle, On transversally simple knots, to appear in J. Differential Geom., available as: math.GT/9910170, 1999.

[CG97] S. Chmutov and V. Goryunov, Polynomial invariants of Legendrian links and their fronts, KNOTS '96 (Tokyo), World Sci. Publishing, River Edge, NJ, 1997, pp. 239-256. 
[Cro89] P. R. Cromwell, Homogeneous links, J. London Math. Soc. (2) 39 (1989), no. $3,535-552$.

[Dun99] N. M. Dunfield, Alexander and Thurston norms of fibred 3-manifolds, to appear in: Pacific J. Math., 1999, available as: math.GT/9908050.

[Fer00] E. Ferrand, On Legendrian knots and polynomial invariants, preprint, available as: math.GT/0002250, 2000.

[FT97] D. Fuchs and S. Tabachnikov, Invariants of Legendrian and transverse knots in the standard contact space, Topology 36 (1997), no. 5, 1025-1053.

[FW87] J. Franks and R. F. Williams, Braids and the Jones polynomial, Trans. Am. Math. Soc. 303 (1987), 97-108.

[Gab83] D. Gabai, Foliations and the topology of 3-manifolds, J. Differential Geom. 18 (1983), no. 3, 445-503.

[Kan98] Y. Kanda, On the Thurston-Bennequin invariant of Legendrian knots and the non exactness of Bennequin's inequality, Invent. Math. 133 (1998), 227-242.

[KL97] K. H. Ko and S. J. Lee, Genera of some closed 4-braids., Topology Appl. 78 (1997), no. 1-2, 61-77.

[Lic88] W.B.R. Lickorish, Polynomials for links, Bull. Lond. Math. Soc. 20 (1988), $558-588$.

[Mam85] N. Mamas, Nudos y tapas, Castillian Math. J 43 (1985), no. 2, 5 -63 (Castillian).

[McM99] C. T. McMullen, The Alexander polynomial of a 3-manifold and the Thurston norm on cohomology, preprint, Harvard University, 1999.

[MH96] H. R. Morton and J. Hodgson, Maple program to compute the multivariable Alexander polynomial, available at: http://www.liv.ac.uk/PureMaths, 1996.

[Mor86] H. R. Morton, Seifert circles and knot polynomials, Math. Proc. Camb. Phil. Soc. 99 (1986), 107-109.

[Mor99] H. R. Morton, The multivariable Alexander polynomial of a closed braid, Lowdimensional topology (H. Nencka, ed.), Contemporary Mathematics, vol. 233, Amer. Math. Soc., 1999, pp. 251-256.

[NW73] C.O.F. Nussbaum and F.E.E. Wu, Gesammelte Schriften zur Theorie der Funktionen einer Veränderlichen, W. Hempel Verlag, Leipzig, 1973 (German).

[Oer86] U. Oertel, Homology branched surfaces: Thurston's norm on $H_{2}\left(M^{3}\right)$, Lowdimensional topology and Kleinian groups (Coventry/Durham, 1984), Cambridge Univ. Press, Cambridge, 1986, pp. 253-272.

[Rud93] L. Rudolph, Quasipositivity as an obstruction to sliceness, Bull. Amer. Math. Soc. (N.S.) 29 (1993), no. 1, 51-59.

[Rud99] L. Rudolph, Positive links are strongly quasipositive, Proceedings of the Kirbyfest (Berkeley, CA, 1998), Geom. Topol., Coventry, 1999, pp. 555-562 (electronic). 
[ST89] M. Scharlemann and A. Thompson, Link genus and the Conway moves, Comment. Math. Helv. 64 (1989), no. 4, 527-535.

[Sta78] J. Stallings, Construction of fibred knots and links, Proc. Symp. Pure Math., vol. 32, AMS, 1978, Part 2, pp. 55-59.

[Tab97] S. Tabachnikov, Estimates for the Bennequin number of Legendrian links from state models for knot polynomials, Math. Research Letters 4 (1997), 143-156.

[Thu86] W. P. Thurston, A norm for the homology of 3-manifolds, Mem. Amer. Math. Soc. 59 (1986), no. 339, i-vi and 99-130.

[Tur75] V. G. Turaev, The Alexander polynomial of a three-dimensional manifold, Mat. Sb. (N.S.) 97(139) (1975), no. 3(7), 341-359, 463.

[Vog90] P. Vogel, Representation of links by braids: A new algorithm, Comm. Math. Helv. 65 (1990), 104-113.

[Xu92] P. Xu, The Genus of Closed 3-Braids, J. Knot Theory and its Ram. 1 (1992), no. $3,303-326$.

University of California, Riverside, Department of Mathematics

Riverside, CA 92521 - 0135, USA

and

Barnard College/Columbia University, Department of Mathematics

New York, NY 10027, USA

Email: kasten@math.ucr.edu and mangum@math.columbia.edu

URL: http://www.math.ucr.edu/^kasten

Received: 14 December 2000 Revised: 21 May 2001 